\title{
NANO-PARTICLES TRANSPORT IN A CONCENTRIC ANNULUS: A LATTICE BOLTZMANN APPROACH
}

\author{
HAMOON POURMirzaAgha \\ Department of Mechanical and Aerospace Engineering, Ramsar Branch, Islamic Azad University, Ramsar, Iran; \\ e-mail: h.poormirzaagha@sina.kntu.ac.ir \\ Hamid Hassanzadeh Afrouzi \\ Young Researchers Club, Babol Branch, Islamic Azad University,Babol, Iran; e-mail: hamidhasanzade@yahoo.com
} Abbasali Abouei Mehrizi

Young Researchers and Elite Club, Karaj Branch, Islamic Azad University, Karaj, Iran; e-mail: abbasabouei@gmail.com

\begin{abstract}
A combination of the lattice Boltzmann method and lagrangian Runge-Kutta procedure is used to study dispersion and removal of nano-particles in a concentric annulus. The effect of aspect ratio, Rayleigh number and particles diameter are examined on particles removal and their dispersion characteristics. Simulations are performed for the Rayleigh number ranges from $10^{3}$ to $10^{5}$ and aspect ratio of 2,3 and 4 . Higher aspect ratios have led to weaker recirculation strength. The finest particles move on stochastic path due to the effect of Brownian motion. The Brownian motion has a greater effect on the removal of nano-particles with respect to thermophoresis.
\end{abstract}

Keywords: LBM, nano-particles, dispersion, removal, Brownian motion, thermophoresis

\section{Introduction}

Natural convection heat transfer in an enclosure occurs in various industrial and engineering applications. Many investigations have been done to study these phenomena in different situations. Heat exchangers as cooling systems in reactors and electrical components of heating and cooling system are some of these examples (Abouei Mehrizi et al., 2013; Jourabian et al., 2013). Dispersion, deposition and removal of solid particles are considered as important cases in numerous industrial, environmental and biological applications. Heat transfer enhancement (Hassanzadeh and Farhadi, 2013; Abouei Mehrizi et al., 2013), air quality (Chen et al., 2006) are some others challages in environmental and biological systems. Studies show that deposition micro-particles and nano-particles on cooling or heating surfaces can improve the systems and significantly increases energy consumption (Rahman et al., 2006; Vessakosol and Charoensuk, 2010). Golovin and Putnam (1962) studied the deposition efficiency of ribbon particles in potential flows. Vasak et al. (1992) investigated solid particles deposition on channel walls in various ranges of flow conditions. One of the major issues in a severe accident scenario is accurate prediction of the deposition rate in adition of understanding and quantifying the removal mechanisms of micro size aerosol particles in buoyancy driven flows inside containments. Puragliesi et al. (2011) investigated micro-particle deposition in the turbulent buoyancy driven flow in a DHC (differentially heated cavity). They employed DNS (Direct Numerical Simulation) for flow field simulation. Their results showed that the largest influence on micro-particles deposition is caused by the gravity effect in such a situation. They discussed the contribution of different forces on the removal of micro-particles in details. Akbar et al. (2009) focused on the behavior of micro-particles in a laminar free convection in a square enclosure. A numerical analysis was performed by Golkarfard et al. (2012) to investigate the transport and deposition 
of aerosols in convection flow in a cavity in the presence of built-in heated obstacles and a driven lid. They analyzed the effect of thermophoresis on deposition of particles, and their result showed that thermophoresis decreases the deposition. The authors performed some researches on the transport of micro-particles in a forced convection flow (Hassanzadeh Afrouzi et al., 2012b) and buoyancy driven flow (Hassanzadeh Afrouzi et al., 2012) employing the lattice Boltzmann method. Annular shapes are applicable geometry in engineering and industry. The geometry of the circular annulus is found in transmission cables, solar collectors-receivers, vapor condenser, heat exchangers. Kuehn and Goldstein $(1976,1978)$ reviewed studies on the free convection in an annulus. It is very important to reach accurate characteristics of the flow field at particle position to calculate the lagrangian particle tracking procedure. The lattice Boltzmann method $(\mathrm{LBM})$ is a suitable numerical technique based on kinetic theory for modeling the physics of fluids systems (Abouei Mehrizi et al., 2012, 2013). In the most recently published papers, the analyzes were done on micro-particles, in which the effects such as the Brownian motion and thermophoresis are not substantial contributors to the particle diffusion mechanism. There is also a lack of understanding of the contribution of specific forces.

In the present study, the contribution of Brownian motion and thermophoresis on the transport of nano-particles is discussed by analysing some case without considering the Brownian motion force and some cases without considering both the Brownian motion and thermophoresis. The lattice Boltzmann method is used to simulate the laminar flow in a concentric annulus. Then the particle equation of motion is solved in the lagrangian framework to investigate the dispersion and removal of particles. Drag, Saffman lift, gravity, buoyancy, Brownian motion and thermophoresis are forces that included in the particle tracking procedure.

\section{Fluid flow simulation}

\subsection{Lattice Boltzmann method}

A two-dimensional laminar natural convection flow is considered in a concentric annulus as the computational domain (Fig. 1).

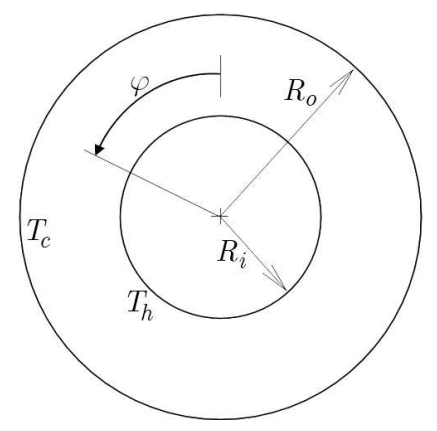

Fig. 1. Computational domain of the concentric annulus

The two-dimensional lattice Boltzmann method is used for flow simulations. A general form of the lattice Boltzmann equation with an external force can be written as (Hassanzade et al., 2012)

$$
f_{k}\left(\mathbf{x}+\mathbf{c}_{k} \Delta t, t+\Delta t\right)-f_{k}(\mathbf{x}, t)=\Delta t \frac{f_{k}^{e q}(\mathbf{x}, t)-f_{k}(\mathbf{x}, t)}{\tau}+\Delta t \cdot \mathbf{F}_{k}
$$

where $f_{k}$ is the distribution function in the direction $k, \Delta t$ is the lattice time step, $\mathbf{c}_{k}$ is the lattice velocity in the direction $k, F_{k}$ is the external force in the direction of $\mathbf{c}_{k}, \tau$ denotes the lattice relaxation time which is defined as $\tau=\left(1 / c_{s}^{2}\right) v+1 / 2$, where $v$ is viscosity, $f_{k}^{e q}$ is 
the equilibrium distribution function dependent on the type of the problem. The equilibrium distribution functions for the fluid field are calculated (Hassanzade et al., 2012) with eguation

$$
f_{k}^{e q}=\omega_{k} \rho\left[1+\frac{\mathbf{c}_{k} \cdot \mathbf{u}}{c_{s}^{2}}+\frac{1}{2} \frac{\left(\mathbf{c}_{k} \cdot \mathbf{u}\right)^{2}}{c_{s}^{4}}-\frac{1}{2} \frac{\mathbf{u} \cdot \mathbf{u}}{c_{s}^{2}}\right]
$$

where $\omega_{k}$ are weighting factors which $k$ changes from 0 to 8 . The values of $\omega_{0}=4 / 9$ for $\left|c_{0}\right|=0$, $\omega_{1-4}=1 / 9$ for $\left|c_{1-4}\right|=1$ and $\omega_{5-8}=1 / 36$ for $\left|c_{5-8}\right|=\sqrt{2}$ are assumed in this model (Abouei Mehrizi et al., 2012). $\rho$ and $\mathbf{u}$ are macroscopic fluid density and velocity which are calculated respectively as below

$$
\rho=\sum_{k} f_{k} \quad \rho \mathbf{u}=\sum_{k} f_{k} \mathbf{c}_{k}
$$

The thermal lattice Boltzmann equation can be written as below

$$
g_{k}(\mathbf{x}, t+\Delta t)-g_{k}(\mathbf{x}, t)=\Delta t \frac{g_{k}^{e q}(\mathbf{x}, t)-g_{k}(\mathbf{x}, t)}{\tau_{c}}
$$

where the thermal equilibrium distribution functions are given as (Hassanzade et al., 2012)

$$
g_{k}^{e q}=\omega_{k} T\left(1+\frac{\mathbf{c}_{k} \cdot \mathbf{u}}{c_{s}^{2}}\right)
$$

where $T$ is the fluid temperature evaluated from

$$
T=\sum_{k} g_{k}^{e q}
$$

The temperature relaxation time is calculated as a function of the diffusivity coefficient

$$
\tau_{c}=\frac{1}{c_{s}^{2}} \alpha+\frac{1}{2}
$$

In order to incorporate the buoyancy force in the model, the Boussinesq approximation has been applied, therefore the force term in Eq. (2.1) needs to be calculated as below in the vertical direction $y$

$$
\mathbf{F}_{y}=3 \omega_{k} \mathbf{g}_{y} \beta \Delta T
$$

where $\mathbf{g}_{y}$ is the acceleration of gravity acting in the $y$-direction of the lattice links, $\beta$ is the thermal expansion coefficient and $\Delta T$ is the temperature difference.

\subsection{Curved boundary treatment}

In the present study, a second-order accurate method to define the curve boundary condition is used (Mei et al., 1999). This boundary condition is used to implement the no slip condition for hydrodynamic and thermal treatment. The curve boundary for the no slip condition is used for both inner and outer cylinders. Figure 2 shows the lattice node treatment on the curved boundary condition, the gray nodes indicate the boundary nodes $x_{b}$, the white nods show the first fluid nodes $x_{f}$, and the solid block nodes on the boundary $x_{w}$ indicate the intersections of the wall with various lattice links (Hassanzadeh Afrouzi et al., 2012).

The fraction of an intersected link in the fluid region $D$, is determined by (Abouei Mehrizi et al., 2012)

$$
D=\frac{\left|x_{f}-x_{w}\right|}{\left|x_{f}-x_{b}\right|}
$$




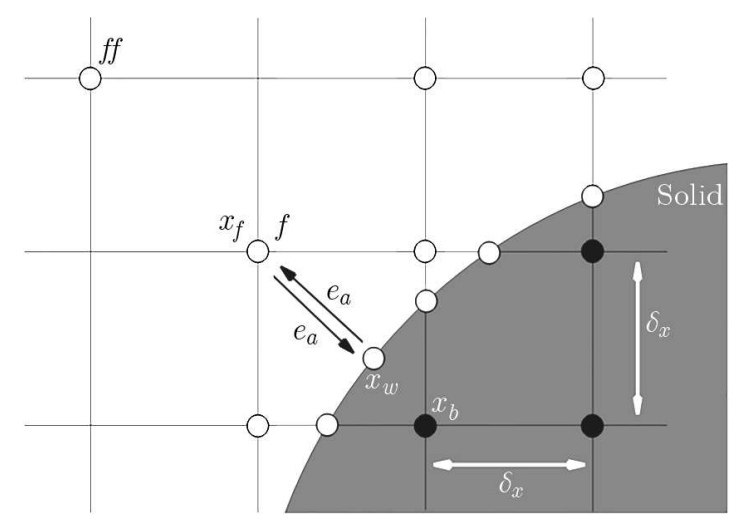

Fig. 2. Layout of the regularly spaced lattices and the curved wall boundary

At the collision step, the fluid side distribution function on the fluid side $\widetilde{f}_{k}$ is determined, but the solid side distribution function at the opposite direction $\widetilde{f}_{\bar{k}}$ is unknown. On the other hand, to finish the streaming step we need to know $\widetilde{f}_{\bar{k}}$ at the boundary node $x_{b}$. The detailed description of calculating velocity and temperature on boundary nodes was presented in our previous work (Hassanzadeh Afrouzi et al., 2012) based on the ref. by Yu et al. (2003) and Guo et al. (2002)

\section{Particle equation of motion}

A particle suspended in a buoyancy driven flow is affected by some forces. The drag, Saffman lift, gravity, buoyancy, Brownian motion and thermophoresis are included in the particle equation of motion in this study. The corresponding particle equation of motion in the $i$-th direction is given as

$$
\frac{d u_{p}^{i}}{d x^{i}}=\frac{1}{\tau_{p}}\left(u_{g}^{i}-u_{p}^{i}\right)+L^{i}+N^{i}+\left(1-\frac{1}{S}\right) g^{i}+T h^{i}
$$

The first term in the right hand side of equations (3.1) is the drag force that is due to relative velocity between the particles and carrier gas. The relaxation time $\tau_{p}$ is the characteristic time scale (response time) of a particle. $u_{p}^{i}$ and $u_{g}^{i}$ are the corresponding particle and velocity in the direction of $i, L$ is Saffman lift force, $N$ - Brownian force, $S$ is specific gravity, $g_{i}$ is gravity acceleration and $T h^{i}$ is the thermophoresis force. By increasing the relaxation time, the particle reaction decreases with variations of flow parameters. For submicron particles with the particle Knudsen number $\mathrm{Kn}_{p}$ larger than 0.1, when the particle diameter is in the range of the gas mean free path $\lambda$, the flow slips over the particle surface. Therefore, the Stokes drag must be modified by the Cunningham correction factor $C_{c}$ as (Shams et al., 2000)

$$
C_{c}=1+\frac{2 \lambda}{d_{p}}\left[1.257+0.4 \mathrm{e}^{\left(\frac{-1.1 d_{p}}{2 \lambda g}\right)}\right]
$$

Small particles in a shear field experience a force perpendicular to the direction of flow. The shear lift originates from the inertia effects in the viscous flow around the particle and is different from the aerodynamic lift force. Saffman (1965) was the first to obtain an equation for this force

$$
L=1.615 \rho^{p} \sqrt{\nu}\left(d^{p}\right)^{2}\left(u^{g}-u^{p}\right) \sqrt{\left|\frac{d u^{f}}{d y}\right|} \operatorname{sgn}\left(\frac{d u^{f}}{d y}\right)
$$

Respectively, $\rho^{p}$ and $\nu$ are density and kinematic viscosity of the gas phase. Brownian motion in the direction $i$ is denoted by $N^{i}$. The instantaneous random momentum imparted to the 
ultrafine particles is due to impacts of gas molecules which make the particle move on an erotic path known as the Brownian motion (Shams et al., 2000; Li and Ahmadi, 1992)

$$
N=G \sqrt{\frac{\pi S_{0}}{d t}} \quad S_{0}=\frac{216 \nu K_{1} T_{g}}{\pi^{2} \rho_{p}\left(d_{p}\right)^{5} S^{2} C_{c}}
$$

where $T_{g}$ is gas temperature and $K_{1}$ is the Boltzmann constant. $G$ is the unit variance zero mean Gaussian random numbers

$$
G=\sqrt{-2 \ln U_{i}} \cos \left(2 \pi U_{j}\right)
$$

where $U_{i}$ and $U_{j}$ are random numbers (between 0 and 1). Finally, $T h^{i}$ denotes thermophoresis force which is defined as

$$
T h^{i}=K_{T h} \frac{\partial T}{\partial x^{i}}
$$

where $K_{T h}$ is the thermophoresis coefficient suggested by Talbot et al. (1980)

$$
K_{T h}=\frac{2 C_{s} C_{c}\left(\frac{K_{g}}{K_{p}}+C_{t} \mathrm{Kn}_{p}\right)}{\left(1+3 C_{m} \mathrm{Kn}_{p}\right)\left(1+2 \frac{K_{f}}{K_{p}}+2 C_{t} \mathrm{Kn}_{p}\right)}
$$

The values of $C_{t}, C_{m}$ and $C_{s}$ are 2.18, 1.14 and 1.17, respectively (Chein and Liao, 2005). $K_{f}$ and $K_{p}$ are thermal conductivity of the fluid and particle, respectively. By solving the particle equation of motion, the particles path is obtained from

$$
\frac{d x^{i}}{d t}=u_{p}^{i}
$$

\section{Numerical procedure}

The computational domain is considered to be two dimensional concentric cylindrical annulus with inner radius $R_{i}$ and outer radius $R_{o}$. The aspect ratio is defined as the ratio of the outer radius to the inner radius. The cylinder surfaces are maintained at two different uniform temperatures. The simulation performed for the Rayleigh number (Ra), Eq. (4.1), from $10^{3}$ to $10^{5}$ and the aspect ratio from 2 to 4 . The characteristics of the annulus are presented in Table 1

$$
\mathrm{Ra}=\frac{g \beta \Delta T H^{3}}{\nu_{g} \alpha_{g}}
$$

Table 1. Annulus characteristics at $\Delta t=100$ and $R_{o}=0.5 \mathrm{~cm}$

\begin{tabular}{|c|c|}
\hline Aspect ratio (AR) & Inner cylinder diameter $[\mathrm{cm}]$ \\
\hline \hline 2 & 0.25 \\
\hline 3 & 0.167 \\
\hline 4 & 0.125 \\
\hline
\end{tabular}

Particle physical properties are listed in Table 2. The Runge-Kutta method is used to calculate particles trajectories. Particles are considered to be captured by surfaces when their distance from the cylinder surfaces becomes smaller than their radius. 
Table 2. Physical properties of particles (silicon dioxide)

\begin{tabular}{|l|c|}
\hline Density $\left[\mathrm{kg} / \mathrm{m}^{3}\right]$ & 2220 \\
\hline Specific heat $[\mathrm{J} /(\mathrm{kg} \mathrm{K})]$ & 745 \\
\hline Thermal conductivity $[\mathrm{W} /(\mathrm{m} \mathrm{K})]$ & 1.38 \\
\hline
\end{tabular}

\section{Results and discussion}

\subsection{Flow field and heat transfer}

Two horizontal circular cylinders are considered. $R_{i}, R_{o}, T_{h}, T_{c}$ stand for the inner and outer radius, temperature of the annulus, respectively. The flow field and heat transfer characteristics are validated by comparing the present results with those by Hauf and Grigull (1966) at the Grashof numbers of 120000 and 122000 . The results show good compatibility between the present simulation and experimental results as shown in Fig. 3. For further validation of the numerical procedure, the local equivalent thermal conductivity is calculated at Ra of $5 \cdot 10^{4}$. The results have been compared with the study by Kuehn and Goldstein (1978). An equivalent thermal conductivity, $K_{e q}$ is used to compare the accuracy of the present computations. The average equivalent heat conductivity is defined for the inner and outer cylinder by

$$
\bar{K}_{e q i}=-\frac{\ln (r r)}{\pi(r r-1)} \int_{0}^{\pi} \frac{\partial T}{\partial r} d \varphi \quad \bar{K}_{e q o}=-\frac{r r \ln (r r)}{\pi(r r-1)} \int_{0}^{\pi} \frac{\partial T}{\partial r} d \varphi
$$
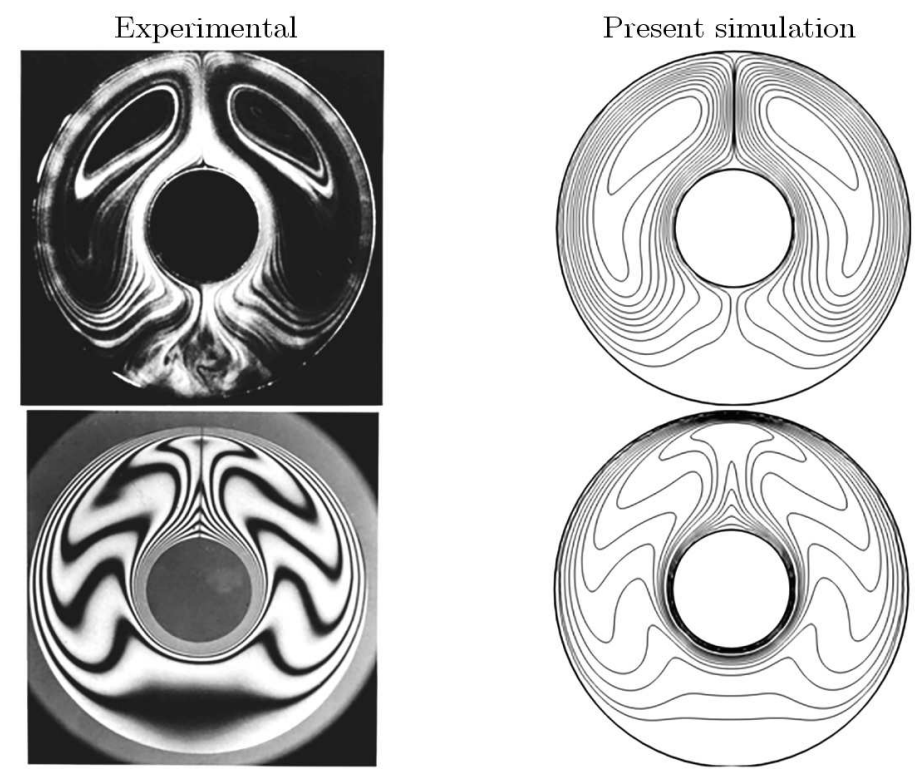

Fig. 3. The streamlines for $\mathrm{Gr}=120000$ (top) and isotherms for $\mathrm{Gr}=122000$ (bottom). Comparison with the experimental result by Hauf and Grigull (1966)

This parameter is defined as the actual heat flux divided by the heat flux that would occur due to pure conduction in the absence of fluid motion. The computed average equivalent heat conductivities are compared with the previous study by Kuehn and Goldstein (1978). The results for the local equivalent thermal conductivity are shown in Fig. 4 and represent good agreement. In particular, the present calculation of the local equivalent thermal conductivity is within $\pm 3 \%$ of their benchmark data. The effect of the annulus aspect ratio on the flow and thermal fields is shown with streamlines (on the left) and the isotherms (on the right) in Figs. 5 to 7, respectively, for three Ra numbers $\left(10^{3}, 10^{4}\right.$ and $\left.10^{5}\right)$. The flow near the hot smaller cylinder becomes hotter 
and reduces density (increase buoyancy). Then near the cold outer cylinder it becomes cold and starts to move down. This formss two large recirculation zones in the annulus at the left and right sides. The flow moves counterclockwise at the left side and clockwise at the right side of annulus. For the smallest Rayleigh number, the heat transfer is mainly due to conduction since the driven fluid motion is very slow (Fig. 5) due to the buoyancy force. As the Rayleigh number increases, the natural convection effect grows up. So the core of recirculation zones stretches in a larger region. The cores additionally move to the upper section of the annulus and this results in a plume shape of the isotherms. The effect of the increasing Ra number is more sensible at higher $\mathrm{AR}(\mathrm{AR}=4$ in Figs. 5 to 7 ). In the bottom section, the relative position of hot and cold walls causes the power of convection to decrease. It is due to that the upper wall in this region is hotter and does not apply any movement to the flow. The increasing of AR causes isotherms to move to the upper section of the annulus gap at $\mathrm{Ra}=10^{3}$. Furthermore, the recirculation zones have less strength by increasing the aspect ratio. The buoyancy driven flow circulates in a narrow region because of the decreasing gap between the circles at a smaller aspect ratio. Therefore, the power of buoyancy becomes greater.

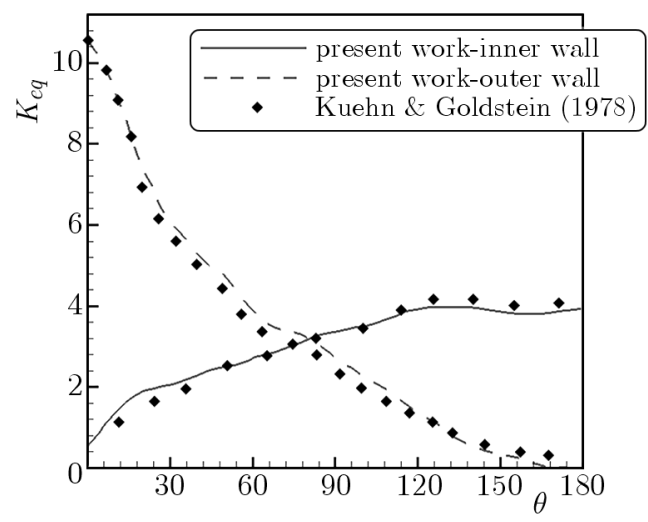

Fig. 4. Equivalent thermal conductivity on the inner and outer cylinder compared with the experiments by Kuehn and Goldstein (1976) for the annulus at $\mathrm{Ra}=5 \cdot 10^{4}$
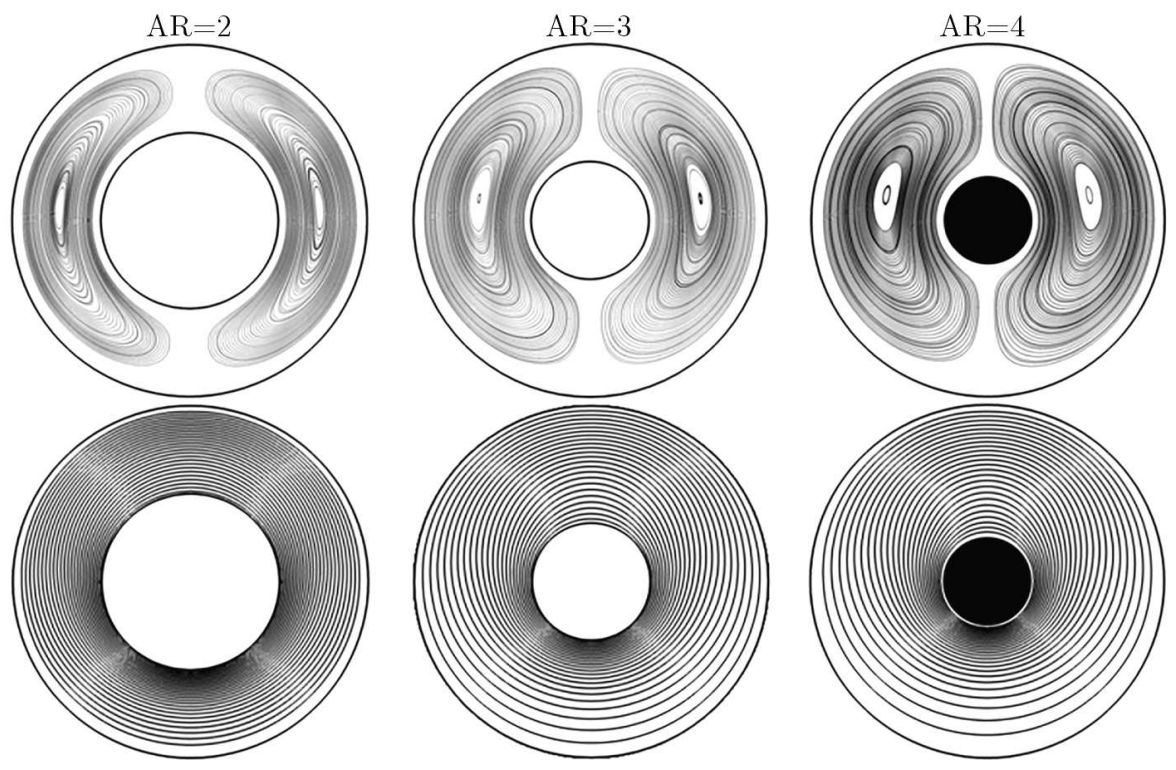

Fig. 5. Streamlines and isotherms for different aspect ratios at $\mathrm{Ra}=10^{3}$ (top: streamlines, bottom: isotherms) 

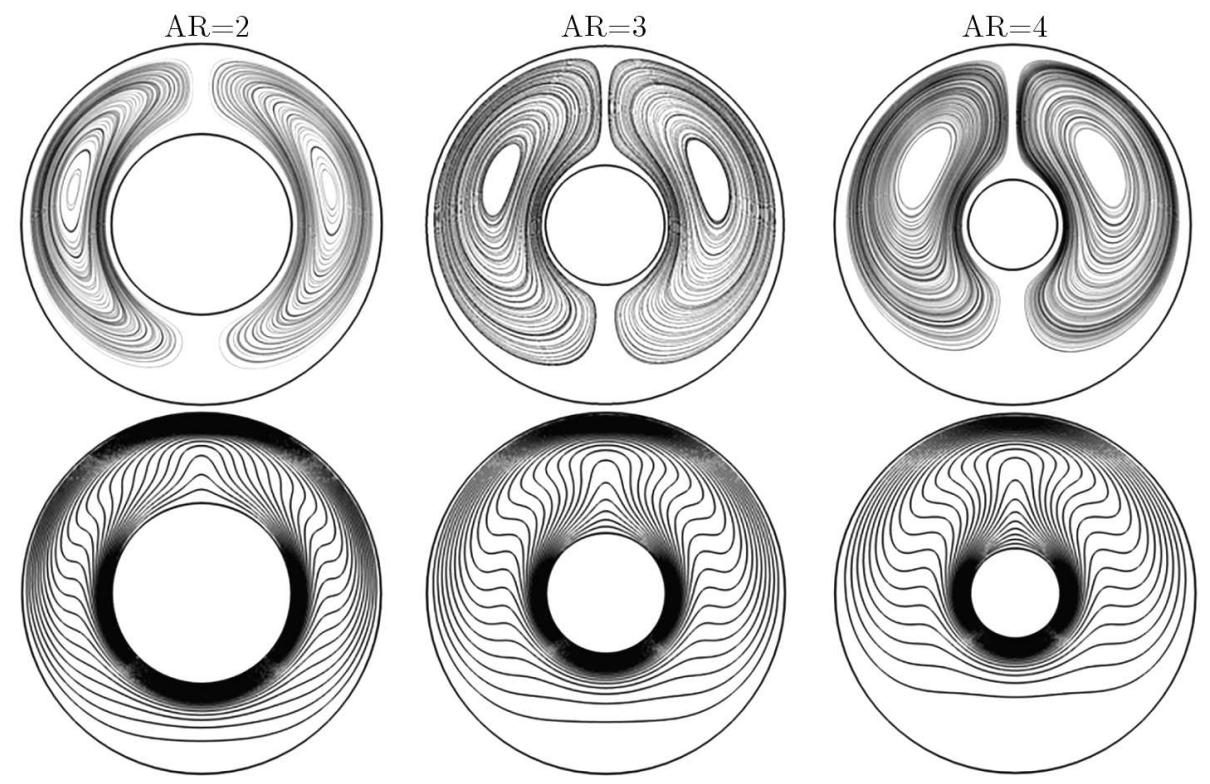

Fig. 6. Streamlines and isotherms for different aspect ratios at $\mathrm{Ra}=10^{4}$ (top: streamlines, bottom: isotherms)
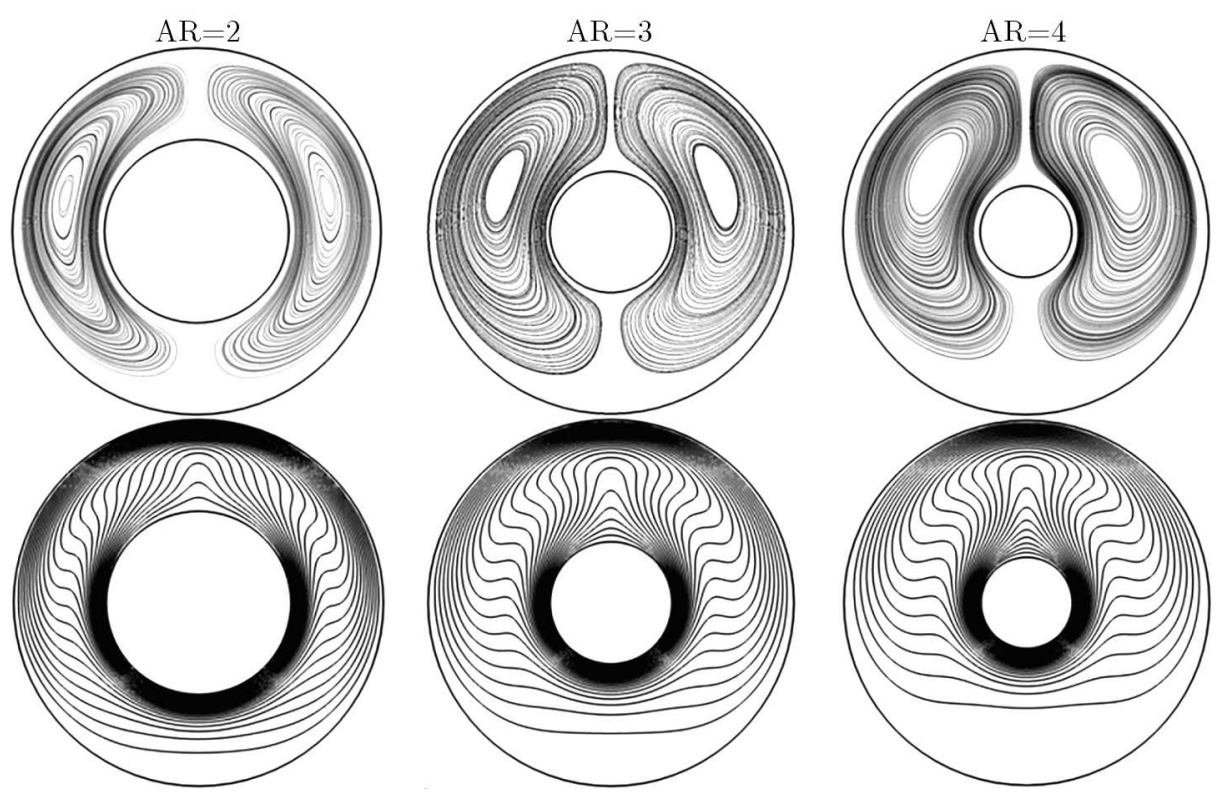

Fig. 7. Streamlines and isotherms for different aspect ratios at $\mathrm{Ra}=10^{5}$ (top: streamlines, bottom: isotherms)

\subsection{Particles dispersion and removal}

In the present study, the particles with sizes in the range of 10 to $150 \mathrm{~nm}$ at $S=2220$ (Golovin and Putnam, 1962) are selected for simulation. The effects of the Rayleigh number, aspect ratio and particles diameter are investigated on the particles behavior such as removal and dispersion. In order to examine the relative significance of various particle transport mechanisms, some cases have been repeated in two new simulation approaches. In the first new approach, the thermophoresis force is neglected and, for the next approach, the effect of both thermophoresis and Brownian motion are not considered. The drag, gravity, buoyancy and lift forces have always been present in all the simulations. Hydrodynamics is usually the primary contributor to the particle dynamics. Since the magnitude and direction of the air velocity in the enclosure is 
changing rapidly, the velocity slip between the air and particle develops. The air flow path is curved and the particles are unable to follow it precisely as a result of inertia. At one point the particles may even hit the wall and they are trapped there. This behavior is known as the inertial impaction. Brownian diffusion is dominant in the removal mechanism for finer particles. However, Brownian motion comes into account for particles with diameter within the range of present simulation.

Two recirculation regions tend to hold the particles within themselves due to hydrodynamic forces. The inertia force pushes the particles outwards and the gravity push them to the bottom. The thermophoretic force drives them toward the negative temperature gradient regions and the Brownian force makes them move along a stochastic path. The simultaneous effect of these forces creates a quasi-equilibrium zone at each side of the annulus gap. The trajectories of some particles are shown in Fig. 8 for $\mathrm{Ra}=10^{3}$ and $\mathrm{AR}=3$ when all forces are applied. The movement of particles with diameter of $10 \mathrm{~nm}$ clearly shows the effect of Brownian motion making the particles path stochastic (Fig. 8a). For these particles, a different removal mechanism is observed. The particles with the ID number of 1 and 2 start their migration from below of hot cylinder where the flow is weaker compared to other regions in the annulus. On the other hand, the power of the Brownian force becomes greater in the hot field. So, these particles are removed from the annulus by Brownian diffusion. The particles with ID numbers of 3 and 4 move through the streamline but the effects of Brownian motion and thermophoresis are observable. The particle with ID number 3 deposits on the inner cylinder by the contribution of Brownian motion and, maybe, gravity. The particles number 4 hit the outer cylinder because of thermophoresis. As the particles become larger, the effect of hydrodynamic forces increases and the particles tend to follow the streamlines exactly. This phenomenon is clearly observed in Figs. 8c and 8d). This figure additionally shows that by increasing the particles diameter from $100 \mathrm{~nm}$ up to $150 \mathrm{~nm}$, no significant changes appear in the manner of particles dispersion in the annulus. It should be noted that the Brownian force causes the particles with diameter of $10 \mathrm{~nm}$ to require more time to follow the flow paths.

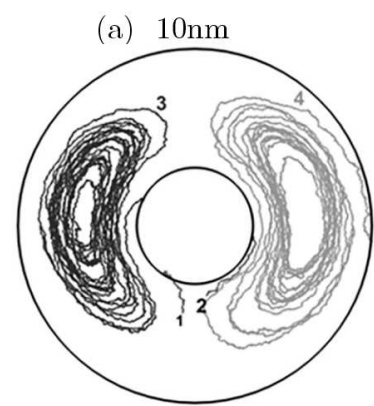

(c) $100 \mathrm{~nm}$

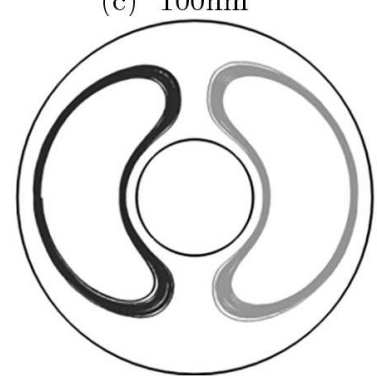

(b) $50 \mathrm{~nm}$

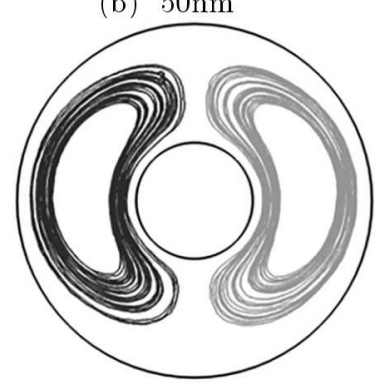

(d) $150 \mathrm{~nm}$

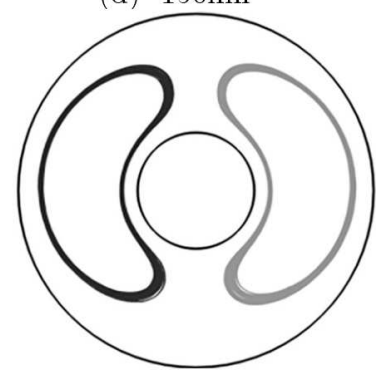

Fig. 8. Particle trajectories with all forces applied for $\mathrm{AR}=3$ and $\mathrm{Ra}=10^{3}$

By increasing the aspect ratio, the recirculation power decreases (Figs. 5 to 7 ), so the particles acceleration and their inertia decrease and the quasi-equilibrium zones cover a large part of the annulus gap (Fig. 8). The instant position of the suspended particles with diameter of $100 \mathrm{~nm}$ 
is shown in Fig. 8 at the Ra number of 10000. It should be noted that the effect of different contributors to the particles transport are very complicated, and the forces have positive or negative effects on one particle in different locations.

The effects of the Ra number and AR of the annulus for particles with different diameters on the particles that remain suspended in the flow (concentration fraction) are presented in Fig. 9. The concentration fraction is smaller at $\mathrm{AR}=4$ for all particles. This shows that the hydrodynamic forces are dependent on the intensity of natural convection flow. By decreasing the aspect ratio, the number of particles that are removed from the flow by hitting the cylinders decreases. Additionally, by increasing the Ra number, the fraction of concentration at constant $\mathrm{AR}$ increases.

(a) $d p=10 \mathrm{~nm}$

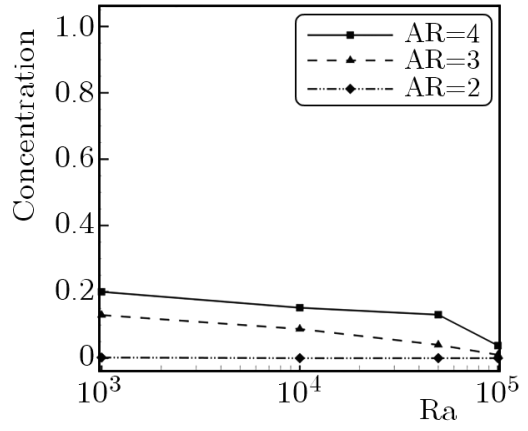

(c) $d p=100 \mathrm{~nm}$

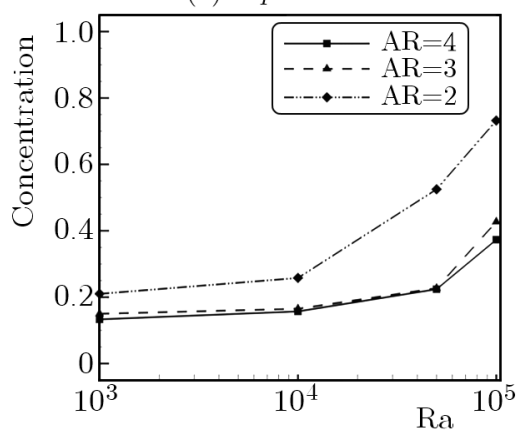

(b) $d p=50 \mathrm{~nm}$

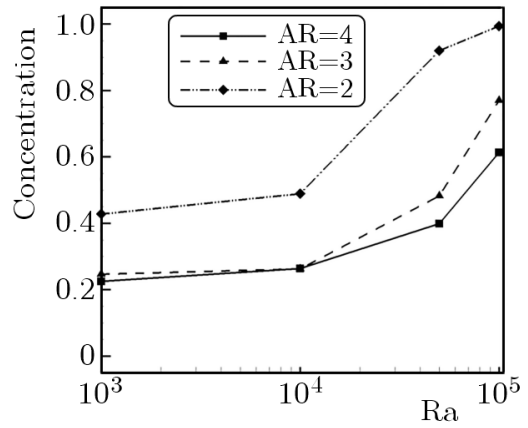

(d) $d p=150 \mathrm{~nm}$

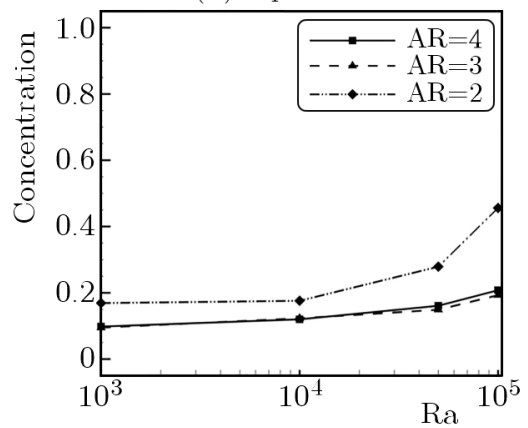

Fig. 9. Concentration fraction for particles with different radii versus the Ra number at various AR

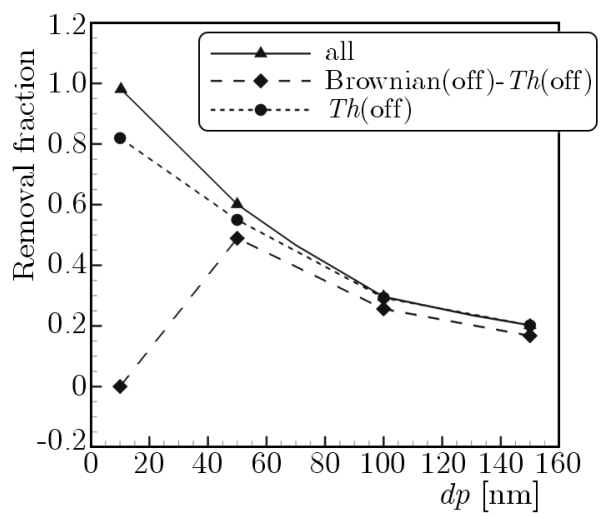

Fig. 10. Concentration versus $d p$ with and without Brownian motion and thermophoresis

The mentioned trend is not observed for the particles with diameter of $10 \mathrm{~nm}$. It is due to domination of Brownian diffusion and also the great effect of thermophoresis on these particles. The particles concentration increases by augmentation of AR. As discussed about the flow results, the quasi equilibrium zones form in a region closer to the cylinder walls at smaller 
$\mathrm{AR}$, and much more particles have chance to diffuse towards both cylinders. The cases with $\mathrm{AR}=0.5$ and $\mathrm{Ra}=10^{4}$ have been repeated two times, one by eliminating the Brownian motion and thermophoresis forces and another by eliminating just the thermophoresis force. The results indicate that the Brownian motion is the dominant removal mechanism, and thermophoresis is the second contributor. As the particles become larger, the effect of Brownian motion decreases while the effect of thermophoresis grows up (Fig. 10). The Brownian motion has no significant effect on the removal of the particles with diameter of $100 \mathrm{~nm}$ and greater. Removal fraction is shown in Fig. 10. It shows the fraction of deposited particles to the total distributed particles in the domain.

\section{Conclusion}

The flow and thermal fileds as well as the nano-particles transport have been investigated numerically by lattice Boltzmann method. The lagrangian particle equation of motion has been solved by employing the 4-th order Runge-Kutta algorithm. Simulations have been performed for the Rayleigh number changing from $10^{3}$ to $10^{5}$ and the particles specific density of 2220 . Two experimental studies have been selected to validate the flow field and thermal characteristic of natural convection. The results show excellent agreement with them. The effects of the cylinder gap and particles size have been investigated on the removal and dispersion of particles at different Rayleigh numbers. Furthermore, the effect of absence and presence of the Brownian and thermophoresis force has been investigated for different cases. The results can be concluded as follows:

- The buoyancy effect increases by augmentation of the Rayleigh number at each aspect ratio and by decreasing the aspect ratio for each Rayleigh number. For a greater buoyancy effect, the strength of recirculation zones decreases.

- Brownian motion causes finer particles to follow stochastic pathlines in the natural convection flow.

- By decreasing the aspect ratio, more particles remove from the flow by hitting the cylinders.

- The final concentration of particles increases by augmentation of the aspect ratio.

- Brownian motion has a stronger more effect on the particles removal for greater nano-particles than the thermophoresis.

Acknowledgement

The present study is supported by the Ramsar branch, Islamic Azad University, Iran.

\section{References}

1. Abouei Mehrizi A., Farhadi M., Hassanzadeh Afrouzi H., Sedighi K., Rabienataj A.A., 2012, Mixed convection heat transfer in a ventilated cavity with hot obstacle: Effect of nano fluid and outlet port location, International Communications in Heat and Mass Transfer, 39, 1000-1008

2. Abouei Mehrizi A., Farhadi M., Hassanzadeh Afrouzi H., Shayamehr S., 2013a, Lattice Boltzmann simulation of natural convection flow around a horizontal cylinder located beneath an insulation plate, Journal of Theoretical and Applied Mechanics, 51, 360-367

3. Abouei Mehrizi A., Farhadi M., Sedighi K., Aghajani Delavar M., 2013b, Effect of fin position and porosity on heat transfer improvement in a plate porous media heat exchanger, Journal of the Taiwan Institute of Chemical Engineers, 44, 3, 420-431 
4. Abouei Mehrizi A., Farhadi M., Shayamehr S., 2013c, Natural convection flow of Cu-Water nanofluid in horizontal cylindrical annuli with inner triangular cylinder using lattice Boltzmann method, International Communications in Heat and Mass Transfer, 44, 3, 147-156

5. Abouei Mehrizi A., Sedighi K., Farhadi M., Sheikholeslami M., 2013d, Lattice Boltzmann simulation of natural convection heat transfer in an elliptical-triangular annulus, International Communications in Heat and Mass Transfer, 48, 164-177

6. Akbar M.K., Rahman M., Ghiansiann S.M., 2009, Particle transport in a small square enclosure in laminar natural convection, Journal of Aerosol Science, 40, 747-761

7. Chein R., Liao W., 2005, Thermophoretic effects on nano-particle deposition in channel flow, Heat and Mass Transfer, 42, 71-79

8. Chen F., Yu S.C.M., Lai A.C.K., 2006, Modeling particle distribution and deposition in indoor environments with a new drift-flux model, Atmospheric Environment, 40, 357-367

9. Golkarfard V., Gandjalikhan Nasab S.A., Ansari A.B., Bagheri G.H., 2012, Numerical investigation on deposition of solid particles in a lid-driven square cavity with inner heated obstacles, Advanced Powder Technology, 23, 6, 736-743

10. Golovin M., Putnam A., 1962, Inertial impaction on single elements, Industrial and Engineering Chemistry Fundamentals, 1, 264-273

11. Guo Z., Shi B., Zheng C., 2002, A coupled lattice BGK model for the Boussinesq equations, International Journal for Numerical Methods in Fluids, 39, 325-342

12. Hassanzadeh Afrouzi H., Farhadi M., 2013, Mix convection heat transfer in a lid driven enclosure filled by nanofluid, Iranica Journal of Energy and Enviornment, 4, 4, 376-384

13. Hassanzadeh Afrouzi H., Farhadi M., Abouei Mehrizi A., 2012a, Numerical simulation of particles transport in a concentric annulus, Advanced Powder Technology, 24, 3, 575-584

14. Hassanzadeh Afrouzi H., Sedighi K., Farhadi M., Fattahi E., 2012b, Dispersion and deposition of micro particles over two square obstacles in a channel via hybrid lattice Boltzmann method and discrete phase model, International Journal of Engineering, 25, 3, 257-266

15. Hauf W., Grigull U., 1966, Measurements of the heat transfer at a horizontal cylindrical container - essential parameters, Waerme- und Stoffuebertragung, 9, 1, 21-28

16. Jourabian M., Farhadi M., Rabienataj A.A., Abouei Mehrizi A., 2013, Lattice Boltzmann simulation of melting phenomenon with natural convection from an eccentric annulus, Thermal Science, 17, 3, 877-890

17. Kuehn T., Goldstein R., 1976, An experimental and theoretical study of natural convection in the annulus between horizontal concentric cylinders, Journal of Fluid Mechanics, 74, 695-719

18. Kuehn T., Goldstein R., 1978, An experimental study of natural convection heat transfer in concentric and eccentric horizontal cylindrical annuli, Journal of Heat Transfer, 100, 635-644

19. Li A., Ahmadi G., 1992, Dispersion and deposition of spherical particles from point sources in a turbulent channel flow, Aerosol Science and Technology, 16, 209-226

20. Mei R., Luo L.S., Shy W., 1999, An accurate curved boundary treatment in the lattice Boltzmann method, Journal of Computational Physics, 155, 307-330

21. Puragliesi R., Dehbi A., Leriche E., Soldati A., Deville M.O., 2011, DNS of buoyancydriven flows and Lagrangian particle tracking in a square cavity at high Rayleigh numbers, International Journal of Heat and Fluid Flow, 32, 915-931

22. Rahman M.Md., Elias Md., Alim M.A., 2009, Mixed convection flow in a rectangular ventilated cavity with a heat conducting square cylinder at the center, Journal of Engineering and Applied Sciences, 4, 5, 155-161

23. SAfFMAn P.G., 1965, The lift on a small sphere in a slow shear flow, Journal of Fluid Mechanics, 22, 385-400 
24. Shams M., Ahmadi G., Rahimzadeh H., 2000, A sublayer model for deposition of nano-and micro-particles in turbulent flows, Chemical Engineering Science, 55, 6097-6107

25. Talbot L., Cheng R., Schefer R., Willis D., 1980, Thermophoresis of particles in a heated boundary layer, Journal of Fluid Mechanics, 101, 737-758

26. Vasak F., Bowen B., Chen C., Kastanek F., Epstein N., 1995, Fine particle deposition in laminar and turbulent flows, Canadian Journal of Chemical Engineering, 73, 785-792

27. Vessakosol P., Charoensuk J., 2010, Numerical analysis of heat transfer and flow field around cross-flow heat exchanger tube with fouling, Applied Thermal Engineering, 30, 1170-1178

28. Yu D., Mei R., Luo L.S., Shyy W., 2003, Viscous flow computations with the method of lattice Boltzmann equation, Progress in Aerospace Sciences, 39, 329-367

Manuscript received November 9, 2014; accepted for print February 24, 2015 